\title{
No Externalities: A Characterization of Efficiency and Incentive Compatibility with Public Goods*
}

\author{
Joseph M. Ostroy ${ }^{\dagger}$ Uzi Segal $^{\ddagger}$
}

September 22, 2010

\begin{abstract}
We show that efficient anonymous incentive compatible (dominant strategy) mechanisms for public goods eliminate externalities, i.e., each individual is unable to change the welfare of anyone else. The characterization is used to derive existence and non-existence results for models with a finite number of individuals and to explain existence results in the continuum. A similar characterization and conclusions are demonstrated for private goods in [7]. However, unlike private goods, elimination of externalities with public goods implies that individuals cannot change the outcome. Hence, such mechanisms provide only weak incentives for truth-telling.
\end{abstract}

KEYWORDS: Public goods; private goods; no externalities; incentive compatibility; duality

JEL Classifications: C72 (Noncooperative Games); D62 (Externalities); D70 (Collective Decision-making); H41 (Public Goods)

\footnotetext{
${ }^{*}$ This project was developed in discussions with Louis Makowski. We thank two referees for the comments.

${ }^{\dagger}$ Department of Economics, UCLA (ostroy@ucla.edu)

$\ddagger^{\ddagger}$ Department of Economics, Boston College (segalu@bc. edu)
} 


\section{Introduction}

We examine the problem of allocating public goods produced by a private good. It is impossible to satisfy efficiency, anonymity, and incentive compatibility (see $[1],[13],[16],[17])$. Our aim is to understand why, by characterizing the demands imposed by these conditions. Our main result is that the mutual consistency of these demands requires the elimination of a literal interpretation of "externalities" — changes in one individual's preferences should not impose utility consequences on anyone else. This holds both for ordinal and quasilinear preferences.

The goal of mechanism design is to find an outcome function (a mechanism) satisfying certain conditions, or to show that no such function exists. For the purposes of this paper, we distinguish between the minimum requirements on the domain of an outcome function such that anonymity, efficiency, and incentive compatibility can be meaningfully defined and the robustness of such conditions. Robustness is typically built into mechanism design by assuming the domain is a Cartesian product of preference profiles. Regarding minimum requirements as separate from robustness will be essential, below.

We consider the set of outcome functions (i.e., mechanisms) defined at a utility profile and ask if there is a neighborhood of this profile (domain of the mechanism) over which an outcome function has the desired features. Efficiency is a pointwise property for each preference profile. Incentive compatibility requires that the neighborhood should include any single-person change in the preference profile. Anonymity is acknowledged by allowing the neighborhood to include profiles where all individuals can report the same preferences. This leads to the construction of "minimally comprehensive" neighborhoods on which the requirements of efficiency, incentive compatibility, and anonymity can be said to be well-defined. We show that on such neighborhoods the desired outcome function must not allow any individual to change the welfare of anyone else (Theorem 2). ${ }^{1}$

To further establish our claim we do two more things. First, we show that there are non-trivial profiles and neighborhoods for which outcomes functions with the desired properties exist. Such neighborhoods can be constructed around an economy such

\footnotetext{
${ }^{1}$ In one direction, this characterization is immediate: an efficient mechanism that eliminates externalities satisfies incentive compatibility. Suppose an individual enjoys a certain utility level, say $\bar{u}$, from reporting his preferences truthfully. If the individual were to misrepresent his preferences, the no externalities condition says that others' utilities would not change. Therefore, if the misrepresentation led to a utility level greater than $\bar{u}$, i.e., was not incentive compatible, that would contradict efficiency. Hence, the goal is to show that an anonymous efficient incentive compatible mechanism must eliminate externalities.
} 
that indifference curves of at least some individuals have "kinks," i.e., discontinuities in marginal rates of substitution. (See Section 4). Second, to prove that ours is really a characterization of outcome functions that are efficient, anonymous, and incentive compatible, we show that an outcome function with the desired properties defined on a domain containing a minimally comprehensive neighborhood would also exhibit no externalities. (Theorem 3).

These conclusions rely on the separation of minimal conditions from robustness. Because externalities are typically unavoidable for efficient outcome functions, our characterization yields impossibility conclusions on domains that are much smaller than a Cartesian product of preference profiles. (Corollary 2) Therefore, one might question the characterization as merely a sharper impossibility result in disguise. To dispel this interpretation we point to the following:

- The characterization has a meaningful economic interpretation. Internalization of externalities has long been regarded as important to harmonize self-interest with efficiency - each person should bear the cost imposed or receive the benefit conferred on others by his actions.

Our findings demonstrate that the modern, mechanism design formalization of this problem confirms the necessity of a literal application of this principle. That its realization is problematic is simply a reflection of the fact that externalities cannot typically be eliminated.

A second reason why the no externalities condition is meaningful is that it is not limited to public goods. The results in [7] show:

- The requirement that externalities must be eliminated holds for the characterization of efficiency, anonymity and incentive incentive compatibility for private goods.

When there are private goods, neighborhoods are created around preference profiles with "flats," i.e., marginal rates of substitution that are constant in a neighborhood.

With public goods, no externalities implies no change in quantities. With private goods, no externalities implies no change in prices: no individual can have a utility consequential effect on the trading opportunities of anyone else, i.e., the environment should be perfectly competitive.

The juxtaposition of no change in quantities versus no change in prices mirrors the well-known duality between public and private goods. ${ }^{2}$ I.e., with private goods,

\footnotetext{
${ }^{2}$ The same duality underlies the relation between flats in convex sets and kinks in their associated support functions.
} 
prices are the "public goods," whereas with public goods, prices are the "private goods." These connections provide added confirmation that the same no externalities principle underlies possibility - and therefore impossibility — in both environments. As a unified summary, the elimination of externalities requires that individuals are unable to change the values of the relevant public goods, i.e., quantities in the case of public goods and prices in the case of private goods.

A third justification concerns robustness.

- No externalities underlies the existence of efficient, incentive compatible, and anonymous outcome functions for populations with large numbers of individuals.

The no externalities characterization described above applies to a small population, i.e., with $n$ individuals. We show that the aggregate kink needed for possibility does not depend on $n$. For fixed $n$, there are many profiles of kinked utilities, each defined on a separate neighborhood, such that within each neighborhood the desired conditions obtain. As $n$ goes to infinity the size of any individual's kink can go to zero, and in the limit all utilities can be differentiable. Consequently, the exceptional character of the no externalities property is more likely as $n$ approaches infinity and it becomes typical for populations in the continuum. Such an argument is demonstrated in [7] for private goods where the size of the flats can be made arbitrarily small as the number of individuals increases and, in the limit, indifference surfaces can be curved. We shall provide a brief sketch, below, of a parallel construction for public goods implying that there are outcome functions that are generically efficient, anonymous, and incentive compatible in economies with a continuum of agents. For results with similar conclusions see [4],[9],[11],[6].

The contrast between the absence of robust existence of anonymous, efficient, incentive compatible outcome functions for small populations and the presence of robust existence with large numbers can be imputed to the absence of robustness of the no externalities property for small populations along with the presence of that property for large populations.

The paper is organized as follows. In Section 2 we define our neighborhoods. The main results are presented in section 3. Section 4 analyzes conditions under which an outcome function has the desired properties and explains why typically it does not exist. Section 5 links the paper to the literature. All proofs appear in the appendix. 


\section{Preliminaries}

Society is composed of $n$ consumers. Person $i=1, \ldots, n$ has preferences $\succeq_{i}$ over pairs $(a, b) \in A \times \Re_{+}$, where $A \subset \Re^{k}$ is a compact convex set of public good vectors and $b$ is a private good. It is assumed throughout that preferences are continuous, convex, and increasing in $b$. The cost of producing public goods is measured in units of the private good and is given by the convex function $\varphi: A \rightarrow \Re_{+}$. Society is endowed with $\bar{b}$ units of the private good which are sufficient to produce any quantities of the public goods in $A$. The set of feasible allocations is

$$
\mathcal{F}=\left\{\left(a, b_{1}, \ldots, b_{n}\right):\left(a, b_{i}\right) \in A \times \Re_{+}, i=1, \ldots, n, \sum_{i} b_{i}+\varphi(a)=\bar{b}\right\}
$$

In the special case of costless public goods $\varphi \equiv 0$.

Preferences are representable by utilities. A mechanism is a function that picks a point in $\mathcal{F}$ according to the social profile of preferences. To simplify notation, we define the domain of the mechanism to be utility vectors and assume that two ordinally equivalent utility vectors lead to the same result. Formally, let $\mathcal{U}$ be the set of continuous, quasiconcave functions, increasing in $b$ but not necessarily differentiable and not necessarily increasing in $a$. In addition, we assume that preferences are restricted to exhibit bounded rates of substitution between public goods and the private good. That is, there exists $M$ such that for all $u$ and $(a, b) \in A \times[0, \bar{b}]$, and $(\Delta a, \Delta b) \neq 0$ such that $u(a, b)=u(a+\Delta a, b+\Delta b)$,

$$
|\Delta b|<M\|\Delta a\|
$$

We refer to this as a Lipschitz condition on $u$. Likewise, we assume that $\varphi$ is Lipschitz (with the same $M$ ). That is, for $\Delta a \neq 0$,

$$
|\varphi(a+\Delta a)-\varphi(a)|<M\|\Delta a\|
$$

Bounds on rates of substitution between public goods and the private good and on the rate of change of the cost function limit the extent to which one individual will be able to influence the outcome. The bound will also ensure the existence of prices (see Prop. 1 in the appendix below). Note that the Lipschitz condition is consistent with a possible absence of differentiability in $u$ and $\varphi$.

We define the domain of the mechanism $f \in \mathcal{F}$ to be elements of $\mathcal{U}^{n}$, with the following restrictions:

Ordinal Equivalence If $\mathbf{u}=\left(u_{1}, \ldots, u_{n}\right)$ and $\mathbf{u}^{\prime}=\left(u_{1}^{\prime}, \ldots, u_{n}^{\prime}\right) \in \mathcal{U}^{n}$ are such that for each $i$ there exists an increasing function $h_{i}$ such that $u_{i}^{\prime}=h_{i} \circ u_{i}$, then $f(\mathbf{u})=f\left(\mathbf{u}^{\prime}\right)$. 
Our analysis is therefore of utility profiles, but each of the assumptions and results to follow can be expressed in terms of preferences only.

For $\mathbf{u}=\left(u_{1}, \ldots, u_{n}\right) \in \mathcal{U}^{n}$ and $u^{\prime} \in \mathcal{U}$, let $\left(\mathbf{u}_{-i}, u_{i}^{\prime}\right)=\left(u_{1}, \ldots, u_{i}^{\prime}, \ldots, u_{n}\right)$. The main features of the mechanism on a domain $\mathcal{D} \subseteq \mathcal{U}^{n}$ are:

Efficiency For every $\mathbf{u} \in \mathcal{D}$, there is no allocation $\left(a, b_{1}, \ldots, b_{n}\right) \in \mathcal{F}$ such that for all $i, u_{i}\left(a, b_{i}\right) \geqslant u_{i}\left(f_{0}(\mathbf{u}), f_{i}(\mathbf{u})\right)$; and for some $j, u_{j}\left(a, b_{j}\right)>u_{j}\left(f_{0}(\mathbf{u}), f_{j}(\mathbf{u})\right)$.

We shall assume that individuals are only identifiable by their characteristics, not their 'names.' Hence, two individuals with the same preferences are indistinguishable and will therefore receive the same allocation of the private, as well as the public, good.

Anonymity If $u_{i}=u_{j}$, then $f_{i}(\mathbf{u})=f_{j}(\mathbf{u})$.

The aim of the paper is to show equivalence of the next three conditions.

Incentive Compatibility ${ }^{3}$ For all $i, \mathbf{u} \in \mathcal{D}, u_{i}^{\prime}$ such that $\left(\mathbf{u}_{-i}, u_{i}^{\prime}\right) \in \mathcal{D}$,

$$
u_{i}\left(f_{0}(\mathbf{u}), f_{i}(\mathbf{u})\right) \geqslant u_{i}\left(f_{0}\left(\mathbf{u}_{-i}, u_{i}^{\prime}\right), f_{i}\left(\mathbf{u}_{-i}, u_{i}^{\prime}\right)\right)
$$

No Externalities For all $i, \mathbf{u} \in \mathcal{D}, u_{i}^{\prime}$ such that $\left(\mathbf{u}_{-i}, u_{i}^{\prime}\right) \in \mathcal{D}$, and $j \neq i$,

$$
u_{j}\left(f_{0}\left(\mathbf{u}_{-i}, u_{i}^{\prime}\right), f_{j}\left(\mathbf{u}_{-i}, u_{i}^{\prime}\right)\right)=u_{j}\left(f_{0}(\mathbf{u}), f_{j}(\mathbf{u})\right)
$$

No Change The mechanism $f$ is constant on $\mathcal{D}$. That is, for all $i, \mathbf{u} \in \mathcal{D}, u_{i}^{\prime}$ such that $\left(\mathbf{u}_{-i}, u_{i}^{\prime}\right) \in \mathcal{D}$,

$$
\left.f_{0}\left(\mathbf{u}_{-i}, u_{i}^{\prime}\right)=f_{0}(\mathbf{u}) \text { and for all } j \text { (including } i\right), f_{j}\left(\mathbf{u}_{-i}, u_{i}^{\prime}\right)=f_{j}(\mathbf{u})
$$

The no externalities condition says that the welfare of any individual $j \neq i$ is invariant to the reported utility of $i$. The no change condition says that the outcome is itself invariant to the reported utility of any individual. Evidently, no change implies no externalities but not conversely. For example, quantities of $a$ and the allocation of its costs may vary such that all individuals are indifferent. In public goods environments this is extremely unlikely. In the domains constructed below for finite numbers of individuals, it does not occur; and the limiting results for large numbers do not rely on it. Theorem 2, below, shows that when $f$ is efficient and

\footnotetext{
${ }^{3}$ Also called strategy-proofness.
} 
anonymous, the following are equivalent: incentive compatibility, no externalities, and no change.

For given utility $u$ and a point $(a, b)$, let $\Psi(a, b ; u)$ be the set of changes that do not reduce the value of $u$. Formally,

$$
\Psi(a, b ; u)=\{(\Delta a, \Delta b): u(a+\Delta a, b+\Delta b) \geqslant u(a, b)\}
$$

Since preferences are convex, $\Psi(a, b ; u)$ is convex. The special case of quasilinearity satisfies the added restriction that $(\Delta a, \Delta b) \in \Psi(a, b ; u)$ if and only if $(\Delta a, \Delta b) \in$ $\Psi(a, b+\alpha ; u)$ for all $\alpha$ such that $b+\alpha \geqslant 0$.

We define the following partial ordering with respect to convex preferences:

Definition 1 The utility function $u^{\prime}$ is a sharpening of $u$ at $(a, b)$ if for $(\Delta a, \Delta b) \neq$ $(0,0)$,

$$
u(a, b)=u(a+\Delta a, b+\Delta b) \Longrightarrow u^{\prime}(a, b)>u^{\prime}(a+\Delta a, b+\Delta b) .
$$

If $u^{\prime}$ is a sharpening of $u$ at $(a, b)$, then except for the common point $(0,0)$, the set of changes for $u^{\prime}, \Psi\left(a, b ; u^{\prime}\right)$, lies entirely inside $\Psi(a, b ; u)$ (see Fig. 1). Sharpening is similar to the comparison used by Maskin [8] to define a condition called monotonicity, except that the strict inequality of eq. (4) is replaced with a weak one. We have not adopted this terminology because sharpening will be used here to introduce the geometry of kinks in convex preferences. We note that the reverse comparison, that we call flattening, plays a parallel role in the characterization of efficiency, anonymity and incentive compatibility with private goods (see [7]).

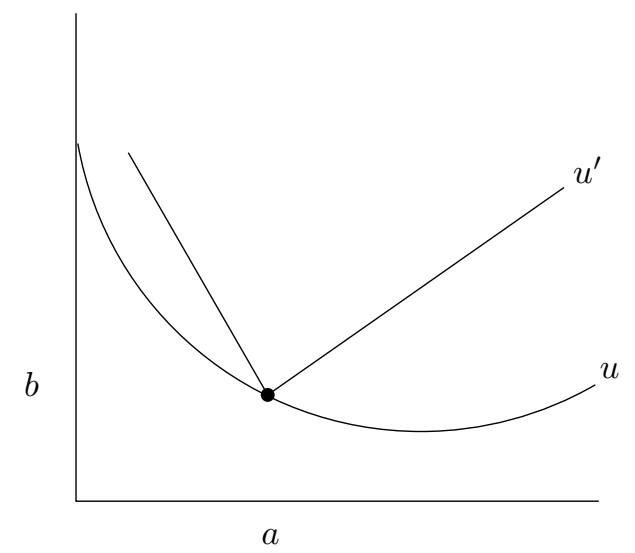

Figure 1: $u^{\prime}$ is a sharpening of $u$ at $(a, b)$. 


\section{Mechanisms}

\subsection{Domains}

Consider a utility profile $\overline{\mathbf{u}}$ and function $f$. Our aim is to check under what conditions one can build a non-trivial domain $\mathcal{D}$ around $\overline{\mathbf{u}}$ such that on this domain $f$ is efficient, anonymous, and incentive compatible, and to analyze the behavior of $f$ on this domain. As mentioned above, mechanism design begins with a domain and looks for a mechanism with given properties whereas we start with the properties and build a domain over which they can be satisfied.

There is a tension between the requirement that the properties can be satisfied and the existence of a meaningful characterization. The smaller the domain the easier it is to satisfy the first requirement. However, if it is too small the requirements will be meaningless. One extreme case is when $\mathcal{D}=\{\overline{\mathbf{u}}\}$ where for all $i \neq j, u_{i} \neq$ $u_{j}$. As utilities are ordinal, there are typically many efficient allocations, and any such allocation will satisfy anonymity (because no two consumers have the same preferences) and incentive compatibility (because no one can declare another utility function). On the other hand, if the domain is too big the assumptions will contradict each other, as on a universal domain no efficient mechanism can be both IC and anonymous (see $[1,13,16,17])$. For the domain to be non-trivial, it should be rich enough so that the above three requirements will be restrictive.

Consider the incentive compatibility condition. We require that at least from the original profile $\overline{\mathbf{u}}$, each individual should be able to announce any type in $\mathcal{U}$. This leads to our first requirement for a domain.

Definition 2 The domain $\mathcal{D}$ is inclusive around $\overline{\mathbf{u}}$ if $\bigcup_{i}\left(\overline{\mathbf{u}}_{-i}, \mathcal{U}\right) \subset \mathcal{D}$.

The fact that $\mathcal{D}$ is inclusive around $\overline{\mathbf{u}}$ is essential. It means that we can apply the full strength of the incentive compatibility at $\overline{\mathbf{u}}$. Note that a domain may be inclusive around several different profiles. If the mechanism were inclusive around every $\mathbf{u} \in \mathcal{D}$, that would imply $\mathcal{D}=\mathcal{U}^{n}$, which leads to an immediate impossibility result as there is no efficient, anonymous, and incentive compatible mechanism on Cartesian products. While our characterization applies to this limiting case, it also applies when the mechanism is only inclusive around some $\overline{\mathbf{u}} \in \mathcal{D}$, a restriction that it critical for making the characterization non-vacuous.

When individuals manipulate their preferences they may potentially affect the allocations of both the public and the private good. As the following lemma shows, incentive compatibility implies no change for the class of possible sharpenings of 
individual utility (see Definition 1). ${ }^{4}$

Lemma 1 Let $\mathbf{u} \in \mathcal{D}$. If $f$ is incentive compatible and $u_{i}^{\prime}$ is a sharpening of $u_{i}$ at $\left(f_{0}(\mathbf{u}), f_{i}(\mathbf{u})\right)$ and $\left(\mathbf{u}_{-i}, u_{i}^{\prime}\right) \in \mathcal{D}$, then $f_{0}\left(\mathbf{u}_{-i}, u_{i}^{\prime}\right)=f_{0}(\mathbf{u})$ and $f_{i}\left(\mathbf{u}_{-i}, u_{i}^{\prime}\right)=f_{i}(\mathbf{u})$.

The implications of anonymity follow from profiles where everyone has the same preferences. One simple way to obtain such profiles is to permit all sharpenings in the domain. Formally, denote by $\mathcal{U}^{*}(u, a, b)$ the set of functions $u^{\prime} \in \mathcal{U}$ where $u^{\prime}$ is a sharpening of $u$ at $(a, b)$. The following definition says that if $\mathbf{u}$ belongs to the domain and the mechanism chooses $f_{0}(\mathbf{u})$, then the domain also includes all sharpenings of $\mathbf{u}$ at $\left(\ldots,\left(f_{0}(\mathbf{u}), f_{i}(\mathbf{u})\right), \ldots\right)$.

Definition 3 The domain $\mathcal{D}$ is first order with respect to $f$ if $\mathbf{u}=\left(u_{1}, \ldots, u_{n}\right) \in \mathcal{D}$ implies that

$$
\prod_{i=1}^{n} \mathcal{U}^{*}\left(u_{i}, f_{0}(\mathbf{u}), f_{i}(\mathbf{u})\right) \subset \mathcal{D} .
$$

We will then say that $(\mathcal{D}, f)$ is first order.

Another property of sharpening of utilities is their benign effect on efficiency. If person $i$ sharpens his utility at the efficient outcome he receives from $f$, the previous Lemma says that $i$ cannot change his outcome and the following Lemma says if the original allocation is efficient, it will continue to be efficient for the perturbed economy.

Lemma 2 Suppose that $f(\mathbf{u})$ is efficient for $u$. Then for all $i$ and $u_{i}^{\prime} \in \mathcal{U}^{*}\left(u_{i}, f_{0}(\mathbf{u})\right.$, $\left.f_{i}(\mathbf{u})\right), f(\mathbf{u})$ is also efficient for $\left(\mathbf{u}_{-i}, u_{i}^{\prime}\right)$.

The first order condition limits the allowable perturbations that individuals can make from any $\mathbf{u} \in \mathcal{D}$, but the fact that they form a Cartesian product says that the first order perturbations for $i$ are independent of those for $j \neq i$. By contrast, the inclusiveness condition consists of the opposite kind of construction. It places no limits on the allowable perturbations any individual can make, but it permits only one-at-a-time departures.

Definition 4 The pair $(\mathcal{D}, f)$ is comprehensive around $\overline{\mathbf{u}}$ if $\mathcal{D}$ is first order with respect to $f$ and $\mathcal{D}$ is inclusive around $\overline{\mathbf{u}}$.

\subsection{Characterization}

A key property of $(\mathcal{D}, f)$ satisfying our assumptions is that the private good must be shared equally. This strong conclusion can be obtained on a very restricted domain

\footnotetext{
${ }^{4}$ Monotonicity/sharpening is used in [8] to establish a similar conclusion with respect to Nash Implementation.
} 
and without assuming that $f$ is efficient. (A similar result is obtained on a larger domain by Barbera and Jackson [1] and Serizawa [13]).

Theorem 1 Let $(\mathcal{D}, f)$ be first order. If $f$ is anonymous and incentive compatible on $\mathcal{D}$, then for every $\mathbf{u} \in \mathcal{D}$ and for every $i$,

$$
f_{i}(\mathbf{u})=\frac{\bar{b}-\varphi\left(f_{0}(\mathbf{u})\right)}{n} .
$$

Among the pairs $(\mathcal{D}, f)$ that are comprehensive around $\mathbf{u}$, where $f_{0}(\mathbf{u})=a^{*}$, we define those that are minimal. By Theorem 1, $f$ allocates the private good equally between all consumers.

(1) Consider all $\mathbf{u}$ where for exactly one $i, u_{i} \in \mathcal{U}$ and for all $j \neq i, u_{j} \in \mathcal{U}^{*}\left(\bar{u}_{j}, a^{*}, \bar{b} / n\right)$. That is, exactly one person moves anywhere, and all other individuals sharpen (or not) their utilities at the outcome they received by the mechanism.

(2) For any $\mathbf{u}$ in $(1)$, if $f_{0}(\mathbf{u}) \neq a^{*}$, then all the economies $\mathbf{u}^{\prime}$ where for all $i$, $u_{i}^{\prime} \in \mathcal{U}^{*}\left(u_{i}, f_{0}(\mathbf{u}), \bar{b} / n\right)$ are also in $(\mathcal{D}, f)$. Such profiles are obtained when some, or all, individuals sharpen their utility at their outcome of the mechanism at profiles obtained by procedure (1).

Condition (1) around $\overline{\mathbf{u}}$ allows any one individual to deviate by announcing any $u_{i}$ among those that are Lipschitz and monotonic in $b$, provided other individuals can only sharpen their utility at their outcome from $f(\mathbf{u})$. By permitting sharpenings at any $\mathbf{u} \in \mathcal{D}$, a first order mechanism (condition (2)) allows individuals to announce other utilities consistent with $f(\mathbf{u})$ being an efficient choice. A minimally comprehensive $(\mathcal{D}, f)$ is the smallest such domain, i.e., with only one $\overline{\mathbf{u}} \in \mathcal{D}$ satisfying the above.

The following is an example of a minimally comprehensive domain.

Example 1 Let $A=[0,1]$ (that is, there is only one public good) and let

$$
\bar{u}_{i}(a, b)= \begin{cases}m_{i} a+b & a \leqslant \frac{1}{2} \\ m_{i}-m_{i} a+b & a>\frac{1}{2}\end{cases}
$$

for $m_{1} \ldots m_{n}>0$ such that $\sum m_{i}>2 M$ (recall that $M$ is the Lipschitz constant). Indifference curves of such utility functions are v-shaped. Assume $\varphi \equiv 0$ and let $\overline{\mathbf{u}}=\left(\bar{u}_{1}, \ldots, \bar{u}_{n}\right)$. By efficiency, $f_{1}(\overline{\mathbf{u}})=\frac{1}{2}$ and by Theorem $1, f_{i}(\overline{\mathbf{u}})=\bar{b} / n, i=$ $1, \ldots, n$. A minimally comprehensive domain $(\mathcal{D}, f)$ around $\overline{\mathbf{u}}$ is the set of all profiles $\mathbf{u}$ where $u_{i^{*}} \in \mathcal{U}$ and for $i \neq i^{*}, u_{i}$ is a sharpening of $\bar{u}_{i}$. That is, for $x<y<\frac{1}{2}$, $u_{i}(y)-u_{i}(x)<-m_{i}(y-x)$ and for $x>y>\frac{1}{2}, u_{i}(x)-u_{i}(y)>m_{i}(x-y), i^{*}=1, \ldots, n$. 
The following is the main result of the paper.

Theorem 2 Let $(\mathcal{D}, f)$ be minimally comprehensive around $\overline{\mathbf{u}} \in \mathcal{U}^{n}$. If $f$ satisfies efficiency and anonymity, then the following three conditions are equivalent: incentive compatibility, no externalities, and no change.

Next, we want to show that our analysis is really a characterization of efficient, incentive compatible mechanisms. We first observe that although we did not assume continuity, since $f$ exhibits no change on $\mathcal{D}$ it is trivially continuous. To establish the uniqueness of the result, we confine our attention to mechanisms that are continuous. Define

Continuity $\mathbf{u}^{\ell} \rightarrow \mathbf{u}$ implies $f\left(\mathbf{u}^{\ell}\right) \rightarrow f(\mathbf{u}) .^{5}$

Suppose that the function $g$ is continuous on $\mathcal{D}$ and $(\mathcal{D}, g)$ is comprehensive with respect to $g$ around $\overline{\mathbf{u}}$. We show that $g$ coincides with $f$.

Theorem 3 Let $(\mathcal{D}, f)$ be comprehensive around $\overline{\mathbf{u}} \in \mathcal{U}^{n}$, where $f$ satisfies continuity, efficiency, anonymity, and incentive compatibility. Suppose that $g$ on $\mathcal{D}$ satisfies continuity, efficiency, anonymity, and either incentive compatibility or no externalities. Then $g \equiv f$.

A remark on anonymity: The key objectives are efficiency and incentive compatibility. Anonymity, too, is a desirable feature: it is also essential. Without it, efficiency and incentive compatibility could be achieved by serial dictatorship in which the most desirable outcome to individual 1 is chosen, and in the case of ties, the most desirable to individual 2 among the remaining, etc.

\section{Possibility and Impossibility Results}

To show that the above characterization is not vacuous, we provide examples of mechanisms and comprehensive domains over which efficiency, anonymity, and incentive compatibility are satisfied. As claimed above, such mechanisms on these environments must exhibit no externalities. To simplify, in this section we restrict attention to the costless case, i.e., $\varphi \equiv 0$.

Let $f_{0}(\overline{\mathbf{u}})=a^{*}$ be the efficient allocation chosen at $\overline{\mathbf{u}}$. From Theorem $1, f_{i}(\mathbf{u})=$ $\bar{b} / n$ for all $\mathbf{u} \in \mathcal{D}$. Recalling that $\mathcal{U}^{*}\left(\bar{u}_{i}, a^{*}, \bar{b} / n\right)$ is the set of sharpening of $\bar{u}_{i}$ at

\footnotetext{
${ }^{5}$ An ordinal definition of continuity should be stated in terms of convergence of preferences. Convergent utilities is a convenient short-cut.
} 
$\left(a^{*}, \bar{b} / n\right)$, let

$$
\mathcal{D}\left(\overline{\mathbf{u}}, a^{*}\right)=\bigcup_{i}\left\{\ldots \mathcal{U}^{*}\left(\bar{u}_{i-1}, a^{*}, \bar{b} / n\right) \times \mathcal{U} \times \mathcal{U}^{*}\left(\bar{u}_{i+1}, a^{*}, \bar{b} / n\right) \times \ldots\right\}
$$

The set $\mathcal{D}\left(\overline{\mathbf{u}}, a^{*}\right)$ contains all the profiles obtained from $\overline{\mathbf{u}}$ by letting one person move anywhere while everyone else is sharpening his utility at his outcome of $f(\overline{\mathbf{u}})$, which by Theorem 1 is $\left(a^{*}, \bar{b} / n\right)$.

For $(\Delta a, \Delta b)$ on the boundary of $\Psi(a, b ; u), \Delta b$ measures the maximum quantity of the private good $b$ an individual with utility $u$ is willing to pay (or the minimum to receive) to make the change $\Delta a$ from the allocation $(a, b)$.

Corollary 1 Let $\overline{\mathbf{u}}$ and $a^{*}$ satisfy for all $\Delta a$ and $\left(\Delta b_{1}, \ldots, \Delta b_{n}\right)$,

$$
\forall i \bar{u}_{i}\left(a^{*}+\Delta a, \bar{b} / n+\Delta b_{i}\right)=\bar{u}_{i}\left(a^{*}, \bar{b} / n\right) \Longrightarrow \sum_{i=1}^{n} \Delta b_{i}>2 M \cdot\|\Delta a\|
$$

Then there is a mechanism $f$ and a domain $\mathcal{D}=\mathcal{D}\left(\overline{\mathbf{u}}, a^{*}\right)$ such that $\mathcal{D}$ is comprehensive around $\overline{\mathbf{u}}$ and $f$ is efficient, anonymous, and incentive compatible on $\mathcal{D}$. For such a mechanism, $f_{0}(\mathbf{u})=a^{*}$ and $f_{i}(\mathbf{u})=\bar{b} / n$ for all $\mathbf{u} \in \mathcal{D}$.

To understand this Corollary, observe first that by Theorem 1, the mechanism should give everyone $\bar{b} / n$ units of the private good. By eq. (6), and given an equal division of the private good, moving from $a^{*}$ to any other allocation $a^{*}+\Delta a$ requires a positive total compensation in terms of the private good, hence $a^{*}$ is the only efficient outcome for $f$ (given an equal division of the private good). Moreover, as the total compensation is more than $2 M \cdot\|\Delta a\|$, it follows that whatever utility function person $i$ chooses, as it is Lipschitz with bound $M$, the total compensation in all directions will still be positive. Person $i$ can therefore not change the efficiency of $\left(a^{*}, \bar{b} / n, \ldots, \bar{b} / n\right)$, and as he cannot change the allocation of the private good he cannot change the allocation and the efficiency of the public goods.

Figure 2 illustrates Corollary 1. Inequality (6) establishes a sufficiently large "kink" at $a^{*}$ among the individuals to nullify the ability of any one person to overturn its efficiency. Even if he were to set the slope of his indifference curve through $\left(a^{*}, \bar{b} / n\right)$ to be $M$ or $-M$.

Remark 1 Note the following.

1. Corollary 1 requires $n \geqslant 3$. Otherwise, inequality (6) cannot be satisfied.

2. The condition in (6) does not imply that for every consumer, or even for any consumer, $a^{*}$ is the optimal quantity for the egalitarian $(\bar{b} / n, \ldots, \bar{b} / n)$ allocation of the private good. (See e.g. consumers 1 and 4 in Fig. 2). 


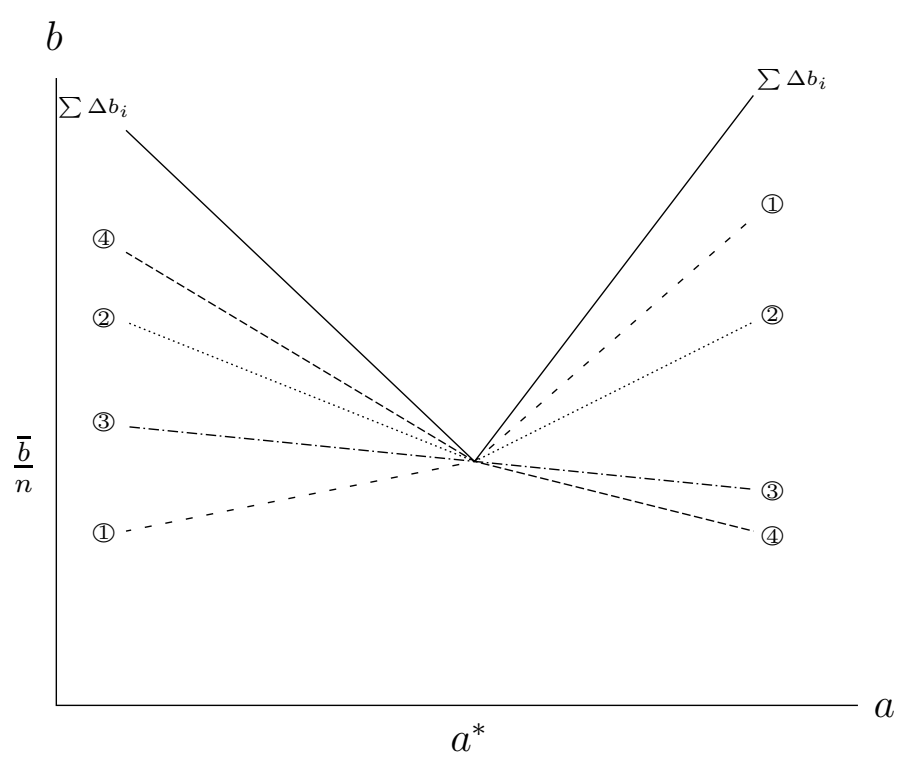

Figure 2: Sum of sharpenings (not drawn to scale)

3. The condition of (6) implies that at least one consumer has nondifferentiable preferences at $a^{*}$, but it does not imply that all consumers have nondifferentiable preferences at this point. (Compare consumers 2 and 3 in Fig. 2. For simplicity, consumer 3's preferences are drawn as linear, but they could be non-linear and differentiable.)

4. The condition in (6) is sufficient, but not necessary. For example, if $n=3$ and $\bar{u}_{1}(a, b)=\bar{u}_{2}(a, b)=\bar{u}_{3}(a, b)=b-4 M\left\|a^{*}-a\right\| / 7$, then the mechanism $f$ such that $f_{0}\left(\overline{\mathbf{u}}_{-i}, u\right)=a^{*}$ and $f_{i}\left(\overline{\mathbf{u}}_{-i}, u\right)=\bar{b} / 3, i=1,2,3$, satisfies all the requirements in $\mathcal{D}\left(\overline{\mathbf{u}}, a^{*}\right)$.

If an efficient mechanism created no externalities, the argument in footnote 1 says that it would be incentive compatible. Conversely, Theorem 2 implies that if a change in the public good allocation were required to accommodate a change in one individual's preferences, that would unavoidably create positive or negative externalities on the payoffs for others. And Theorem 2 also implies that the presence of externalities creates opportunities for strategic manipulation. To show how nongeneric is the possibility of exact efficiency and incentive compatibility with a fixed finite population, the following suffices for impossibility:

Corollary $\mathbf{2}$ If $(\mathcal{D}, f)$ is comprehensive around each $\mathbf{u} \in \mathcal{D}$ and there exists no $a \in A$ such that $f_{0}(\mathcal{D}) \equiv\{a\}$, then $f$ is not incentive compatible and it exhibits externalities. 
To illustrate impossibility, recall that by Theorem $1, b_{i}=\bar{b} / n$ for all $i$. Hence, no consumer is punished in his $b$-good compensation for announcing false preferences. It is easy to construct examples where given the fixed allocation of the $b$-good, $a^{*}=f_{0}(\overline{\mathbf{u}})$ is not the best point for one consumer and this consumer has the ability to change this outcome by announcing false preferences. For example, suppose $k=1$ ( $k$ is the number of public goods), $A=[0,1]$, let $\bar{u}_{2}(a, b)=\ldots=\bar{u}_{n}(a, b)=b-M a / 2 n$, and let $\bar{u}_{1}(a, b)=b+M a / 2 n$. Let $u_{1}(a, b)=\frac{1}{2} M a+b$ to obtain $f_{0}(\overline{\mathbf{u}})=0$ but $f_{0}\left(\overline{\mathbf{u}}_{-1}, u_{1}\right)=1$. So consumer 1 is better off announcing $u_{1}$ instead of his true utility $\bar{u}_{1}$.

The fragility of the possibility result in Corollary 1 is demonstrated by Corollary 2 . Corollary 1 can also be used to point out why the larger the number of individuals, the smaller the influence any one individual has on an efficient choice of public goods. In the limiting case of a continuum of individuals, if the selection of efficient outcomes is a continuous function of the distribution of individual preferences, the influence of any one individual's preferences necessarily vanishes. Hence, the conflict between efficiency, anonymity and the elimination of externalities also vanishes.

As a building block for the asymptotic analysis underlying the results in the continuum, consider the following extension of Corollary 1 that demonstrates possibility on a domain consisting of disjoint unions of minimally comprehensive domains.

Corollary 3 Let $n \geqslant 3$. There is an efficient, anonymous, incentive compatible, no externalities mechanism $f$ on the domain

$$
\begin{array}{r}
\mathcal{D}=\bigcup_{a^{*}}\left\{\mathcal{D}\left(\overline{\mathbf{u}}, a^{*}\right): \overline{\mathbf{u}} \text { and } a^{*} \text { satisfy inequality }(6)\right. \\
\text { and } \left.\mathcal{D}\left(\overline{\mathbf{u}}, a^{*}\right) \text { is minimally comprehensive }\right\}
\end{array}
$$

The disjoint unions of minimally comprehensive domains highlighted in Corollary 3 cover only a small subset of $\mathcal{U}^{n}$. Nevertheless, they suggest how the collection $\bigcup_{a^{*}} \mathcal{D}\left(\overline{\mathbf{u}}, a^{*}\right)$ can come closer to filling out $\mathcal{U}^{n}$ the larger is $n$. Using the techniques employed in [7], the set $\mathcal{U}^{n} \backslash\left\{\bigcup_{a^{*}} \mathcal{D}\left(\overline{\mathbf{u}}, a^{*}\right)\right\}$ applied to an approximate notion of incentive compatibility can be shown to converge to a closed no-where dense set in the continuum limit.

\section{Concluding Comments}

A Post-Samuelson View of Public Goods: Concern about free-rider behavior with respect to public goods was an important precursor of mechanism design. Writing before those ideas had been formalized, Samuelson [12] contrasted private and public goods with respect to the size of the market: 
In ordinary market economics as you increase the number of sellers of a homogeneous commodity indefinitely, you pass from monopoly through indeterminate oligopoly and can hope to reach a determinate competitive equilibrium in the limit. It is sometimes thought that increasing the number of citizens who are jointly supplied public goods leads to a similar determinate result. This is reasoning from a incorrect analogy. A truer analogy in private economics would be the case of a bilateral-monopoly supplier of joint products whose number of joint products - meat, horn, hide, and so on - is allowed to increase without number: such a process does not lead to a determinate equilibrium of the type praised in the literature.

This paper and the results of [7] demonstrate that Samuelson's conjectured conclusions about "indeterminacy" can be rephrased in terms of externalities. In each of the above environments, the necessary and sufficient condition for aligning incentives with efficiency is the elimination of externalities. Whether goods are public or private, externalities are typically unavoidable when the number of individuals is not large. Moreover, the presence of externalities can be interpreted as an indeterminacy with respect to the gains from trade, leading to a tradeoff between efficiency and incentive compatibility. When goods are private, externalities can typically be eliminated when the number of individuals is large, with a consequent determinacy in the gains from trade. Samuelson correctly conjectured that with large numbers and public goods, Lindahl equilibrium allocations would not be incentive compatible. Nevertheless, equal cost-sharing is an anonymous efficient incentive compatible mechanism because with large numbers individuals are typically unable to change the outcome, hence externalities are eliminated. However, unlike private goods, the inability to change the outcome implies that there are no positive rewards associated with incentive compatibility.

The Vickrey-Clarke-Groves Characterization: Our focus on no externalities resembles the conclusions of [15], [2], and [3], called VCG mechanisms. Such schemes need not satisfy anonymity, but that requirement can be added without changing its essential features. More important is the fact that its conclusions are based on the hypothesis that preferences are quasilinear. Quasilinearity allows the allocation of resources to be separated into non-money commodity components and the money commodity. This, in turn, permits a qualified notion of efficiency with respect to non-money allocations that is well-defined even if the allocation of money commodity payments is not consistent with full efficiency, i.e., does not satisfy budget balance with respect 
to the money commodity.

The VCG approach applies on an unrestricted domain of quasilinear preferences. The main result is that to achieve qualified efficiency and incentive compatibility, individuals should internalize the externalities they create. This implies that the money payment associated with the individual's non-money allocation should, up to a constant, equal its social cost as measured by its consequences, positive or negative, on the welfare of others. In principle, this is similar to no externalities. But, in practice it differs because the absence of budget balance means that the payment made by the individual causing the externalities is not necessarily received by those on whom it is imposed. In contrast, by insisting on a characterization of incentive compatibility with full efficiency - where existence is problematic, we are placing the quasilinear model on a similar footing to the ordinal model. This repositioning allows us to demonstrate that the no externalities characterization is equally applicable to both.

No Change: The no change characterization of efficiency, anonymity and incentive compatibility with public goods is also the conclusion of other characterizations involving incentive compatibility. In the context of quasilinear preferences, Schummer [14] extends the notion of strategy-proofness (incentive compatibility) to bribeproofness, defined as the absence of gain to both from one individual paying another to misreport his preferences. He shows that when the domain of preferences is sufficiently rich, e.g., smoothly connected, the only bribe-proof allocations are those precluding change, even when money payments are not required to satisfy budget balance.

The externalities described here are a consequence of a change in an individual's utility caused by a change in the allocation of resources. It does not include those situations in which an individual can report information that changes the utility of a given allocation for another individual, called interdependent values. In a quasilinear model, and also without imposing budget balance, Jehiel et. al. [5] show that on a rich domain allowing for externalities/public goods as well as interdependent values, the only incentive compatible allocations are those precluding change.

Public Goods and Social Choice: If the choice set is one dimensional and preferences are single-peaked, Moulin [10] showed that selecting the most preferred alternative of the median voter is anonymous, efficient and incentive compatible. If preferences are convex, they are single-peaked along any one dimension. Zhou [17] showed that when the dimension of the set of alternatives is greater than one, restricting to continuous and convex preferences implies that the only incentive compatible mechanisms are 
dictatorial. Barbera and Jackson [1] characterize incentive compatible mechanisms that are not necessarily efficient or anonymous.

\section{Appendix: Proofs}

Proof of Lemma 1 Let $\left(a, b_{i}\right)=\left(f_{0}(\mathbf{u}), f_{i}(\mathbf{u})\right)$ and let $\left(a^{\prime}, b_{i}^{\prime}\right)=\left(f_{0}\left(\mathbf{u}_{-i}, u_{i}^{\prime}\right)\right.$, $\left.f_{i}\left(\mathbf{u}_{-i}, u_{i}^{\prime}\right)\right)$. If $u_{i}\left(a^{\prime}, b_{i}^{\prime}\right)>u_{i}\left(a, b_{i}\right)$, then person $i$ with the true utility $u_{i}$ will be better off announcing $u_{i}^{\prime}$. Otherwise, $u_{i}\left(a^{\prime}, b_{i}^{\prime}\right) \leqslant u_{i}\left(a, b_{i}\right)$. If $\left(a, b_{i}\right) \neq\left(a^{\prime}, b_{i}^{\prime}\right)$, then, by the definition of a sharpening, $u_{i}^{\prime}\left(a, b_{i}\right)>u_{i}^{\prime}\left(a^{\prime}, b_{i}^{\prime}\right)$, and person $i$ with the true utility $u_{i}^{\prime}$ will be better off announcing $u_{i}$ instead. This leaves $\left(a, b_{i}\right)$ as the only possible outcome of $f_{i}\left(\mathbf{u}_{-i}, u_{i}^{\prime}\right)$.

Proof of Lemma 2 For $j \neq i$, denote $u_{j}^{\prime}=u_{j}$, and denote $\left(a, b_{1}, \ldots, b_{n}\right)=f(\mathbf{u})$. Suppose that there exists $\left(a^{\prime}, b_{1}^{\prime}, \ldots, b_{n}^{\prime}\right)$ such that for all $j, u_{j}^{\prime}\left(a^{\prime}, b_{j}^{\prime}\right) \geqslant u_{j}^{\prime}\left(a, b_{j}\right)$ and for some $k, u_{k}^{\prime}\left(a^{\prime}, b_{k}^{\prime}\right)>u_{k}^{\prime}\left(a, b_{k}\right)$. But if $u_{i}^{\prime}\left(a^{\prime}, b_{i}^{\prime}\right) \geqslant(>) u_{i}^{\prime}\left(a, b_{i}\right)$, then by the definition of sharpening, $u_{i}\left(a^{\prime}, b_{i}^{\prime}\right) \geqslant(>) u_{i}\left(a, b_{i}\right)$, a contradiction to the assumption that $\left(a, b_{1}, \ldots, b_{n}\right)$ is efficient for $\mathbf{u}$.

Proof of Theorem 1 The proof is based on the method used by Makowski, Ostroy, and Segal [7] and is similar to the one used by Serizawa [13, Prop. 1]. As details vary, we provide a complete proof of the theorem.

Observe first that by the Lipschitz condition (1) and the hypothesis that the domain of $u_{i}$ is the compact set $A \times[0, \bar{b}]$, there exists $M^{\prime}<M$ such that

$$
\left|b^{\prime}-b\right|<M^{\prime}\left\|a-a^{\prime}\right\|
$$

Let $a^{*}=f_{0}(\mathbf{u})$. Since the mechanism utilizes all of $\bar{b}$ (that is, $\sum_{i=1}^{n} f_{i}(\mathbf{u})=$ $\left.\bar{b}-\varphi\left(a^{*}\right)\right)$, it follows that if not all agents receive the same quantity of the $b$-good, then there is some $i$, say $i=1$, such that $f_{1}(\mathbf{u})>\left[\bar{b}-\varphi\left(a^{*}\right)\right] / n$. Define

$$
u^{*}(a, b)=b-M^{\prime}\left\|a-a^{*}\right\|
$$

Clearly, $u^{*}$ is a strict sharpening of all the functions $u_{i}$ at $\left(a^{*}, b\right)$ for all $b$. Let $\mathbf{u}^{1}=\left(\mathbf{u}_{-1}, u^{*}\right)$ and obtain by Lemma 1 that $f_{0}\left(\mathbf{u}^{1}\right)=f_{0}(\mathbf{u})=a^{*}$ and $f_{1}\left(\mathbf{u}^{1}\right)=$ $f_{1}(\mathbf{u})>\left[\bar{b}-\varphi\left(a^{*}\right)\right] / n$.

Since $f_{1}\left(\mathbf{u}^{1}\right)>\left[\bar{b}-\varphi\left(a^{*}\right)\right] / n$ and $\sum_{i=1}^{n} f_{i}\left(\mathbf{u}^{1}\right)=\bar{b}-\varphi\left(a^{*}\right)$, there must be another person, say 2 , such that $f_{2}\left(\mathbf{u}^{1}\right)<\left[\bar{b}-\varphi\left(a^{*}\right)\right] / n$. Let $\mathbf{u}^{2}=\left(\mathbf{u}_{-2}^{1}, u^{*}\right)$. Again by Lemma $1, f_{2}\left(\mathbf{u}^{2}\right)=f_{2}\left(\mathbf{u}^{1}\right)$ and $f_{0}\left(\mathbf{u}^{2}\right)=f_{0}\left(\mathbf{u}^{1}\right)=a^{*}$. But now consumers 1 and 
2 have the same utility function, therefore, by anonymity, both receive the same amount of $b$, which is strictly less than $\left[\bar{b}-\varphi\left(a^{*}\right)\right] / n$. So there is another person, say 3 , such that $f_{3}\left(\mathbf{u}^{2}\right)>\left[\bar{b}-\varphi\left(a^{*}\right)\right] / n$. Continuing to replace the utility functions of all individuals by $u^{*}$ we get at the end that all consumers have the same utility function, but either for all $i, f_{i}>\left[\bar{b}-\varphi\left(a^{*}\right)\right] / n$ ( $n$ is odd), or for all $i, f_{i}<\left[\bar{b}-\varphi\left(a^{*}\right)\right] / n(n$ is even). In both cases we get a violation of $\sum b_{i}=\bar{b}-\varphi\left(a^{*}\right)$.

The proof of Theorem 2 will utilize Prop. 1 below. We defined in eq. (3)

$$
\Psi(a, b ; u)=\{(\Delta a, \Delta b): u(a+\Delta a, b+\Delta b) \geqslant u(a, b)\}
$$

In addition, $(\Delta a, \Delta b)$ on the boundary of $\Psi(a, b ; u)$ satisfies the Lipschitz property (1). Fixing the price of the $b$ good to be 1 , let

$$
\Psi^{*}(a, b ; u)=\{p:(p, 1) \cdot \Psi(a, b ; u) \geqslant 0\}
$$

define the normals to the set of supporting hyperplanes to $\Psi(a, b ; u)$ at $(a, b)$. The convexity and Lipschitz properties of $\Psi(a, b ; u)$ plus the fact that $\Psi(a, b ; u)$ contains the zero element on its boundary imply that $\Psi^{*}(a, b ; u) \neq \varnothing$.

The geometry of production is based on

$$
\Upsilon(a, \varphi(a))=\{(\Delta a, \Delta b): \Delta b \geqslant \varphi(a+\Delta a)-\varphi(a)\} .
$$

Using the same normalization for the $b$ good, let

$$
\Upsilon^{*}(a, \varphi(a))=\{p:(p,-1) \cdot \Upsilon(a, \varphi(a)) \leqslant 0\}
$$

Reflecting its cost, the price of the $b$ good enters with a minus sign. Again, the convexity and Lipschitz properties of $\varphi$ and the fact that the zero element is on its boundary imply that $\Upsilon^{*}(a, \varphi(a)) \neq \varnothing$.

The aggregate as-least-as-good-as change set for $\mathbf{u}$ at $f(\mathbf{u})=\left(f_{0}(\mathbf{u}), f_{i}(\mathbf{u})\right)$ is:

$$
\boldsymbol{\Phi}(f(\mathbf{u}) ; \mathbf{u})=\left\{(\Delta a, \Delta b): \forall i\left(\Delta a, \Delta b_{i}\right) \in \Psi\left(f_{0}(\mathbf{u}), f_{i}(\mathbf{u}) ; u_{i}\right), \sum_{i} \Delta b_{i}=\Delta b\right\} .
$$

Note: the same $\Delta a$ is applied in each $\Psi\left(f_{0}(\mathbf{u}), f_{i}(\mathbf{u}) ; u_{i}\right)$.

The convexity of $\boldsymbol{\Phi}(f(\mathbf{u}) ; \mathbf{u})$ and $\boldsymbol{\Upsilon}\left(f_{0}(\mathbf{u}), \varphi\left(f_{0}(\mathbf{u})\right)\right)$ is well-known to imply that

Proposition 1 (pricing and efficiency) The mechanism $f$ is efficient at $\mathbf{u}$ iff there exists $p \in \mathbf{\Upsilon}^{*}\left(f_{0}(\mathbf{u}), \varphi\left(f_{0}(\mathbf{u})\right)\right.$ and $p_{i} \in \Psi^{*}\left(f_{0}(\mathbf{u}), f_{i}(\mathbf{u}) ; u_{i}\right)$ satisfying $\sum p_{i}=p$ such that

$$
(p,-1) \cdot \Upsilon\left(f_{0}(\mathbf{u}), \varphi\left(f_{0}(\mathbf{u})\right)\right) \leqslant 0 \leqslant \sum_{i}\left(p_{i}, 1\right) \cdot \Psi\left(f_{0}(\mathbf{u}), f_{i}(\mathbf{u}), u_{i}\right)
$$


Equivalently,

$$
0 \in \mathbf{\Phi}^{*}(f(\mathbf{u}) ; \mathbf{u})-\Upsilon^{*}\left(f_{0}(\mathbf{u}), \varphi\left(f_{0}(\mathbf{u})\right)\right.
$$

Proof of Theorem 2 Let $\mathbf{u}, \mathbf{u}^{\prime} \in \mathcal{D}$, and suppose that $f_{0}(\mathbf{u})=a^{*}$ but $f_{0}\left(\mathbf{u}^{\prime}\right)=a^{\prime} \neq$ $a^{*}$. As $\mathcal{D}$ is minimally comprehensive around $\overline{\mathbf{u}}$, we can assume wlg that

1. $a^{*}=f_{0}(\overline{\mathbf{u}})$,

2. there exists $i$ such that for all $j \neq i, u_{j}$ is a sharpening of $\bar{u}_{j}$ at $\left(a^{*},\left[\bar{b}-\varphi\left(a^{*}\right)\right] / n\right)$,

3. $\mathbf{u}^{\prime}=\left(\mathbf{u}_{-i}, u_{i}^{\prime}\right)$.

We deal first with the case of no production cost (that is, $\varphi \equiv 0$ ). By Theorem 1 , for all $j, f_{j}(\mathbf{u})=f_{j}\left(\mathbf{u}^{\prime}\right)=\bar{b} / n$. We assume first that for all $i, u_{i}\left(a^{*}, \bar{b} / n\right) \neq u_{i}\left(a^{\prime}, \bar{b} / n\right)$ and $u_{i}^{\prime}\left(a^{*}, \bar{b} / n\right) \neq u_{i}^{\prime}\left(a^{\prime}, \bar{b} / n\right)$.

The arguments below relate essentially to a line in $\Re^{k}$, so we assume first one public good only. We show at the end of the proof how to extend it to many goods. Assume, wlg, $a^{\prime}<a^{*}$.

First we want to replace, for all $j \neq i$, the function $u_{j}$ with $\tilde{u}_{j}$ which will satisfy the following requirements (See Fig. 3).

1. It will be a sharpening of $u_{j}$ at $\left(a^{*}, \bar{b} / n\right)$;

2. It will be a sharpening of $u_{j}$ at $\left(a^{\prime}, \bar{b} / n\right)$;

3. The slopes of its indifference curves along the $\left[a^{\prime}, a^{*}\right]$ segment will be constant.

As $\sum b_{j}=\bar{b}$ and for all $j, b_{j} \geqslant 0$, the relevant domain over which all utilities are defined is compact. There exists therefore $M^{\prime \prime}<M$ such that for all $j, u_{j}$ satisfies the Lipschitz condition with respect to $M^{\prime \prime}$. Choose $M^{\prime} \in\left(M^{\prime \prime}, M\right)$ and let the slope of all indifference curves of $\tilde{u}_{j}$ be $-M^{\prime}$ to the left of $a^{\prime}$ and $M^{\prime}$ to the right of $a^{*}$. We assumed that for all $j \neq i, u_{j}\left(a^{*}, \bar{b} / n\right) \neq u_{j}\left(a^{\prime}, \bar{b} / n\right)$. Suppose that $u_{j}\left(a^{\prime}, \bar{b} / n\right)<u_{j}\left(a^{*}, \bar{b} / n\right)$. Let $b^{\prime}<\bar{b} / n$ such that $u_{j}\left(a^{\prime}, \bar{b} / n\right)<u_{j}\left(a^{*}, b^{\prime}\right)$ and define the slope of the indifference curves of $\tilde{u}_{j}$ between $a^{\prime}$ and $a^{*}$ to be $-\left(\bar{b} / n-b^{\prime}\right) /\left(a^{*}-a^{\prime}\right)$ The construction of $\tilde{u}_{j}$ for the case $u_{j}\left(a^{\prime}, \bar{b} / n\right)>u_{j}\left(a^{*}, \bar{b} / n\right)$ is similar.

Denote

- $\tilde{\mathbf{u}}=\left(\ldots, \tilde{u}_{i-1}, u_{i}, \tilde{u}_{i+1}, \ldots\right)$;

- $\tilde{\mathbf{u}}^{\prime}=\left(\ldots, \tilde{u}_{i-1}, u_{i}^{\prime}, \tilde{u}_{i+1}, \ldots\right)$; 


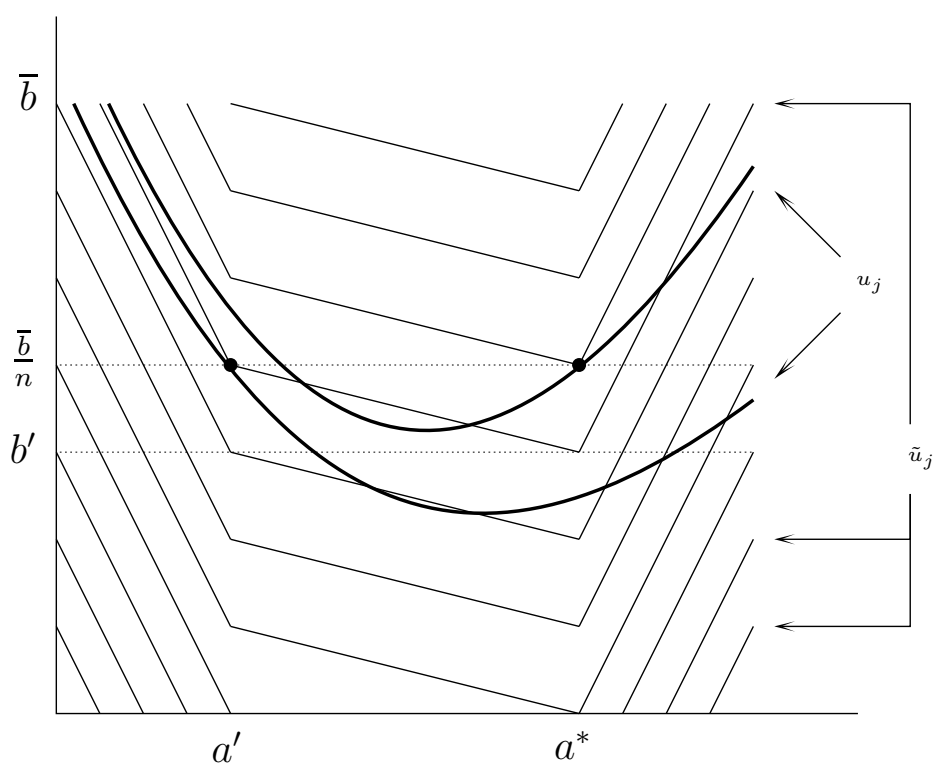

Figure 3: Before and after sharpenings at $a^{\prime}$ and $a^{*}$

By Lemma $1, f_{0}(\tilde{\mathbf{u}})=a^{*}$ and $f_{0}\left(\tilde{\mathbf{u}}^{\prime}\right)=a^{\prime}$. Recall that $a^{\prime}<a^{*}$, and let

- $x=\sum_{j \neq i} \operatorname{MRS}^{b, a}\left(\tilde{u}_{j}(a, \bar{b} / n)\right)$ for $a \in\left(a^{\prime}, a^{*}\right)$;

- $y=\lim _{a \uparrow a^{*}} \operatorname{MRS}^{b, a}\left(u_{i}(a, \bar{b} / n)\right)$;

- $z=\lim _{a \downarrow a^{\prime}} \operatorname{MRS}^{b, a}\left(u_{i}^{\prime}(a, \bar{b} / n)\right)$.

By the construction of $\tilde{u}_{j}, x$ is constant along $\left(a^{\prime}, a^{*}\right)$. By the efficiency of $f_{0}(\tilde{\mathbf{u}})=a^{*}$ and $f_{0}\left(\tilde{\mathbf{u}}^{\prime}\right)=a^{\prime}$, we obtain that $x+y \leqslant 0$ but $x+z \geqslant 0$ (see Prop. 1). Our aim is to show that person $i$ has the ability to manipulate $f_{0}$ to his benefit, a violation of IC.

As $x+y \leqslant 0$ but $x+z \geqslant 0$, and as $|y|,|z|<M^{\prime}$, it follows that $x \in\left(-M^{\prime}, M^{\prime}\right)$. Let $\hat{u}_{i}^{\eta}$ be such that for all $b$,

$$
\operatorname{MRS}^{b, a}\left(\hat{u}_{i}^{\eta}(a, b)\right)=\left\{\begin{aligned}
-M^{\prime} & a<a^{\prime} \\
-\eta & a \in\left(a^{\prime}, a^{*}\right) \\
M^{\prime} & a>a^{*}
\end{aligned}\right.
$$

By Prop. 1, if $\eta+x<0$ [resp., $\eta+x>0$ ], then the only efficient point where the private good is equally allocated is $a^{*}$ [resp., $a^{\prime}$ ]. Therefore, if $x>0$, then person $i$ with the utility $\hat{u}^{-x+\varepsilon}, 0<\varepsilon<\min \left\{M-M^{\prime},|x|\right\}$, can switch the economy from $a^{\prime}$, where it will be if he declares $\hat{u}^{-x+\varepsilon}$, to $a^{*}$, which he favors, by stating $\hat{u}^{-x-\varepsilon}$. 
Similarly, if $x<0$, person $i$ with the true utility $\hat{u}^{-x-\varepsilon}$ can switch the economy from $a^{*}$, where it will be if he declares $\hat{u}^{-x-\varepsilon}$, to $a^{\prime}$, which he favors, by stating $\hat{u}^{-x+\varepsilon}$. If $x=0$, sharpen slightly the utility of one person $j \neq i$ at $a^{*}$ and follow the same analysis.

We assumed that for all $j \neq i, u_{j}\left(a^{*}, \bar{b} / n\right) \neq u_{j}\left(a^{\prime}, \bar{b} / n\right)$. If for some $j \neq i$, $u_{j}\left(a^{*}, \bar{b} / n\right)=u_{j}\left(a^{\prime}, \bar{b} / n\right)$, then it is impossible to satisfy points 1 and 2 in the construction of $\tilde{u}_{j}$. In that case define $\tilde{u}_{j}$ as above, only that between $a^{\prime}$ and $a^{*}$ the slope of the indifference curves will be zero. By construction $a^{*}=f_{0}(\tilde{\mathbf{u}})$ and $a^{\prime}=f_{0}\left(\tilde{\mathbf{u}}^{\prime}\right)$, as these are the only efficient allocations satisfying an equal allocation of the private good.

Our analysis was done with respect to one public good only. We only provide a sketch of a proof for the general case, based on the above analysis. To extend the sharpening of Fig. 3 to $\Re^{k}$, recall that the vertical axis in this figure represents the private commodity and the horizontal axis is the line through $\left(a^{\prime}, 0\right)$ and $\left(a^{*}, 0\right)$. The sharpening in the other directions will be to the slope of $-M^{\prime}$ (or, when necessary, $\left.-M^{\prime \prime}\right)$ towards the indifference curves of the picture.

Lastly, we extend the proof to the case of non-zero production cost. Let $(\mathcal{D}, f)$ be first order and suppose that the mechanism $f$ on $\mathcal{D}$ satisfies efficiency, anonymity, and incentive compatibility when the cost function is $\varphi$. We use this mechanism to create a mechanism $g$ on a first order domain $\mathcal{D}^{\prime}$ that satisfies efficiency, anonymity, and incentive compatibility when there are no production costs.

For every $u \in \mathcal{U}$, define $v(a, b ; u)=u(a, b-\varphi(a) / n)$ and for $\mathbf{u}=\left(u_{1}, \ldots, u_{n}\right) \in \mathcal{U}^{n}$, let $\mathbf{v}(\mathbf{u})=\left(v_{1}(\cdot ; u), \ldots, v_{n}(\cdot ; u)\right)$. Observe that since $u$ and $\varphi$ are Lipschitz, so is $v(\cdot ; u)$ (even if with a different constant). Let $\mathcal{D}^{\prime}=\{\mathbf{v}(\mathbf{u}): \mathbf{u} \in \mathcal{D}\}$, and define a mechanism $g$ for the domain $\mathcal{D}^{\prime}$ by

$$
\begin{aligned}
& \text { - } g_{0}(\mathbf{v}(\mathbf{u}))=f_{0}(\mathbf{u}) ; \text { and } \\
& \text { - } g_{i}(\mathbf{v}(\mathbf{u}))=f_{i}(\mathbf{u})+\frac{\varphi\left(f_{0}(\mathbf{u})\right)}{n}
\end{aligned}
$$

Lemma $3\left(g, \mathcal{D}^{\prime}\right)$ is first order.

Proof For every profile of utilities in $\mathcal{D}^{\prime}$ there is a profile in $\mathcal{D}$ generating it. So let $\mathbf{v}(\mathbf{u}) \in \mathcal{D}^{\prime}$ and let $v_{i}^{\prime}$ be a sharpening of $v_{i}$ at $\left(g_{0}(\mathbf{v}), g_{i}(\mathbf{v})\right)$. Since $g_{0}(\mathbf{v}(\mathbf{u}))=f_{0}(\mathbf{u})$ and $g_{i}(\mathbf{v}(\mathbf{u}))=f_{i}(\mathbf{u})+\frac{\varphi\left(f_{0}(\mathbf{u})\right)}{n}$, the function $u_{i}^{\prime}(a, b):=v_{i}^{\prime}\left(a, b+\frac{\varphi(a)}{n}\right)$ is a sharpening of $u_{i}$ at $\left(f_{0}(\mathbf{u}), f_{i}(\mathbf{u})\right)$. By the comprehensibility of $\mathcal{D}, \mathbf{u}^{\prime}=\left(u_{1}^{\prime}, \ldots, u_{n}^{\prime}\right) \in \mathcal{D}$, and as $\mathbf{v}^{\prime}=\mathbf{v}^{\prime}\left(\mathbf{u}^{\prime}\right), \mathbf{v}^{\prime} \in \mathcal{D}^{\prime}$.

Lemma 4 The mechanism $g$ on $\mathcal{D}^{\prime}$ satisfies efficiency, anonymity, and incentive compatibility. 
Proof The proof follows by the fact that the mechanism $f$ on $\mathcal{D}$ satisfies these three properties.

Efficiency: We utilize here a variant of Proposition 1. When the cost of the public good is given by the convex function $\varphi(a)$, the set $\Upsilon(a, \varphi(a))=\{(\Delta a, \Delta b): \varphi(a+$ $\Delta a)+\Delta b \geqslant \varphi(a)\}$ is convex, as is $\Upsilon^{*}(a, \varphi(a))=\{p:(p, 1) \cdot(\Delta a, \Delta b) \leqslant 0,(\Delta a, \Delta b) \in$ $\Upsilon(a, \varphi(a))\}$. It is well-known that for convex $\Psi$, a mechanism $f$ is efficient at $\mathbf{u}$ iff

$$
0 \in \sum_{i} \Psi^{*}\left(f_{0}(\mathbf{u}), f_{i}(\mathbf{u}) ; u_{i}\right)+\Upsilon^{*}\left(f_{0}(\mathbf{u}), \sum_{i} f_{i}(\mathbf{u})\right)
$$

By definition,

$$
\Psi^{*}\left(g_{0}(\mathbf{v}(\mathbf{u})), g_{i}(\mathbf{v}(\mathbf{u})) ; v_{i}\left(u_{i}\right)\right)=\Psi^{*}\left(f_{0}(\mathbf{u}), f_{i}(\mathbf{u}) ; u_{i}\right)+\frac{1}{n} \nabla(\varphi)
$$

Hence by eq. (7) efficiency is satisfied.

Anonymity: Suppose $v_{i}\left(u_{i}\right)=v_{j}\left(u_{j}\right)$. Then $u_{i}=u_{j}$. Since $f$ satisfies anonymity, $f_{i}(\mathbf{u})=f_{j}(\mathbf{u})$ and $g_{i}(\mathbf{v}(\mathbf{u}))=g_{j}(\mathbf{v}(\mathbf{u}))$.

Incentive Compatibility: Let $\mathbf{v}$ and $\mathbf{v}^{\prime}=\left(\mathbf{v}_{-i}, v^{\prime}\right)$ be in $\mathcal{D}^{\prime}$. So there are $u_{1}, \ldots, u_{n}, u^{\prime}$ such that $\mathbf{u}$ and $\mathbf{u}^{\prime}:=\left(\mathbf{u}_{-i}, u_{i}^{\prime}\right)$ are in $\mathcal{D}, \mathbf{v}=\mathbf{v}(\mathbf{u})$, and $\left.\mathbf{v}^{\prime}=\mathbf{v}^{\prime}\left(\mathbf{u}^{\prime}\right)\right)$. By definition and the incentive compatibility of $f$,

$$
\begin{aligned}
& v_{i}\left(g\left(\mathbf{v}^{\prime}\right)\right)=v_{i}\left(g_{0}\left(\mathbf{v}^{\prime}\right), g_{i}\left(\mathbf{v}^{\prime}\right)\right)= \\
& v_{i}\left(f_{0}\left(\mathbf{u}^{\prime}\right), f_{i}\left(\mathbf{u}^{\prime}\right)+\frac{\varphi\left(f_{0}\left(\mathbf{u}^{\prime}\right)\right)}{n}\right)= \\
& u_{i}\left(f_{0}\left(\mathbf{u}^{\prime}\right), f_{i}\left(\mathbf{u}^{\prime}\right)\right)= \\
& u_{i}\left(f\left(\mathbf{u}^{\prime}\right)\right) \leqslant \\
& u_{i}(f(\mathbf{u}))= \\
& u_{i}\left(f_{0}(\mathbf{u}), f_{i}(\mathbf{u})\right)= \\
& \left.v_{i}\left(f_{0}(\mathbf{u})\right), f_{i}(\mathbf{u})+\frac{\varphi\left(f_{0}(\mathbf{u})\right)}{n}\right)= \\
& v_{i}\left(g_{0}(\mathbf{v}), g_{i}(\mathbf{v})\right)=v_{i}(g(\mathbf{v}))
\end{aligned}
$$

We obtain that $g$ satisfies all the requirements of Theorem 2 for the case $\varphi \equiv 0$, and therefore $g$ exhibits no change mechanism. Since $g_{0}(\mathbf{v}(\mathbf{u}))=f_{0}(\mathbf{u})$, it follows that $f$ too exhibits no change.

Proof of Theorem 3 We prove the theorem for the case of zero production cost. The extension to the general case is similar to the one used in the proof of Theorem 2.

Observe first that by Theorem 2, the minimally comprehensive domain around $\overline{\mathbf{u}} \in \mathcal{U}^{n}$ is $\mathcal{D}(\overline{\mathbf{u}}, f(\overline{\mathbf{u}}))$ (see eq. (5)). Given $\mathbf{u} \in \mathcal{D}(\overline{\mathbf{u}}, f(\overline{\mathbf{u}}))$ we may therefore assume, wlg, that 
(*) For all $i>1, u_{i}$ is a sharpening of $\bar{u}_{i}$ at $\left(f_{0}(\overline{\mathbf{u}}), \bar{b} / n\right)$.

Similarly to Theorem 1, we can prove the following for $g$ :

Fact 1 If for some $\mathbf{u} \in \mathcal{D}(\overline{\mathbf{u}}, f(\overline{\mathbf{u}})), g_{0}(\mathbf{u})=a^{*}:=f_{0}(\overline{\mathbf{u}})$, then for all $i, g_{i}(\mathbf{u})=\bar{b} / n$.

We say that the function $u(a, b)$ is locally quasilinear around $\left(a^{o}, b^{o}\right)$ if there is $\varepsilon>0$, a function $v: A \rightarrow \Re$, and an increasing function $h: \Re \rightarrow \Re$ such that

$$
\left|u(a, b)-u\left(a^{o}, b^{o}\right)\right|<\varepsilon \Longrightarrow h(u(a, b))=v(a)+b
$$

Suppose that for some $\mathbf{u} \in \mathcal{D}(\overline{\mathbf{u}}, f(\overline{\mathbf{u}})), g(\mathbf{u})=a \neq a^{*}=f_{0}(\overline{\mathbf{u}})$. We first create an economy $\mathbf{u}^{\prime}$ such that for all $i$

- $g_{0}\left(\ldots, u_{i}^{\prime}, u_{i+1}, \ldots\right) \neq a^{*}$

- $u_{i}^{\prime}$ is quasiconcave and is a sharpening of $u_{i}$ at $\left(a^{*}, \bar{b} / n\right)$

- $u_{i}^{\prime}$ is locally quasilinear around $\left(a^{*}, \bar{b} / n\right)$

Denote $\mathbf{u}^{i}=\left(\ldots, u_{i}^{\prime}, u_{i+1}, \ldots\right)$. Suppose we created $u_{i-1}^{\prime}$ and construct $u_{i}^{\prime}$.

Case 1. If

$$
u_{i}\left(g_{0}\left(\mathbf{u}^{i-1}\right), g_{i}\left(\mathbf{u}^{i-1}\right)\right)<u_{i}\left(a^{*}, \frac{\bar{b}}{n}\right)
$$

then replace $u_{i}$ with $u_{i}^{\prime}=u^{*}$, given by

$$
u^{*}(a, b)=b-M^{\prime}\left\|a-a^{*}\right\|
$$

for some $M^{\prime} \in\left(M^{\prime \prime}, M\right)$ where $M^{\prime \prime}$ is such that for all $j, u_{j}$ satisfies the Lipschitz condition with respect to $M^{\prime \prime}$. Clearly, $u^{*}$ is quasilinear and a sharpening of $u_{i}$ at $\left(a^{*}, \bar{b} / n\right)$. By fact 1 , if $g_{0}\left(\mathbf{u}^{i}\right)=a^{*}$, then $g_{i}\left(\mathbf{u}^{i}\right)=\bar{b} / n$ and person $i$ benefits from replacing $u_{i}$ with $u_{i}^{\prime}$, a violation of $\mathrm{IC}$, hence $g_{0}\left(\mathbf{u}^{i}\right) \neq a^{*}$.

Case 2. If

$$
u_{i}\left(g_{0}\left(\mathbf{u}^{i-1}\right), g_{i}\left(\mathbf{u}^{i-1}\right)\right)=u_{i}\left(a^{*}, \frac{\bar{b}}{n}\right)
$$

then, using the same technique as in the proof of Theorem 2 (see Fig. 3), replace $u_{i}$ with $u_{i}^{\prime}$ which is a sharpening of $u_{i}$ at $\left(a^{*}, \bar{b} / n\right)$ and at $\left(g_{0}\left(\mathbf{u}^{i-1}\right), g_{i}\left(\mathbf{u}^{i-1}\right)\right)$ such that eq. (9) is satisfied with $u_{i}^{\prime}$. As eq. (9) restricts one indifference curve only, we can construct $u_{i}^{\prime}$ to be quasilinear, and by construction, $u_{i}^{\prime}$ is quasiconcave. The function $u_{i}^{\prime}$ is only a weak sharpening of $u_{i}$ at $\left(g_{0}\left(\mathbf{u}^{i-1}\right), g_{i}\left(\mathbf{u}^{i-1}\right)\right)$, but it is the limit of sharpenings $u_{i, m}^{\prime}$ of $u_{i}$ at $\left(g_{0}\left(\mathbf{u}^{i-1}\right), g_{i}\left(\mathbf{u}^{i-1}\right)\right)$. The functions $u_{i, m}^{\prime}$ are not sharpenings 
of $u_{i}$ at $\left(a^{*}, \bar{b} / n\right)$, but our domain permits one person's utility to be any function in $\mathcal{U}$. If $i=1$, then by condition $(*)$, for all other agent $j$, his utility is a sharpening of $u_{j}$ at $\left(a^{*}, \bar{b} / n\right)$. If $i>1$, then person 1 utility was already replaced by a sharpening of $u_{1}$ at $\left(a^{*}, \bar{b} / n\right)$ (see steps 1 and 3$)$. As $u_{i, m}^{\prime}$ is a sharpening of $u_{i}$ at $\left(g_{0}\left(\mathbf{u}^{i-1}\right), g_{i}\left(\mathbf{u}^{i-1}\right)\right)$, it follows by Theorem 1 that

$$
g_{0}\left(\mathbf{u}_{-i}^{i-1}, u_{i, m}^{\prime}\right)=g_{0}\left(\mathbf{u}^{i-1}\right) \neq a^{*}
$$

and by continuity, $g_{0}\left(\mathbf{u}^{i}\right) \neq a^{*}$.

Case 3. If

$$
u_{i}\left(g_{0}\left(\mathbf{u}^{i-1}\right), g_{i}\left(\mathbf{u}^{i-1}\right)\right)>u_{i}\left(a^{*}, \frac{\bar{b}}{n}\right)
$$

then replace $u_{i}$ with $u_{i}^{\prime}$ which is depicted on Fig. 4 . This function is locally quasilinear around $\left(a^{*}, \bar{b} / n\right)$. It is quasiconcave, and a sharpening of $u_{i}$ at $\left(a^{*}, \bar{b} / n\right)$. It is also a sharpening of $u_{i}$ at $\left(g_{0}\left(\mathbf{u}^{i-1}\right), g_{i}\left(\mathbf{u}^{i-1}\right)\right)$. We thus obtain that $g_{0}\left(\mathbf{u}^{i}\right)=g_{0}\left(\mathbf{u}^{i-1}\right) \neq a^{*}$.

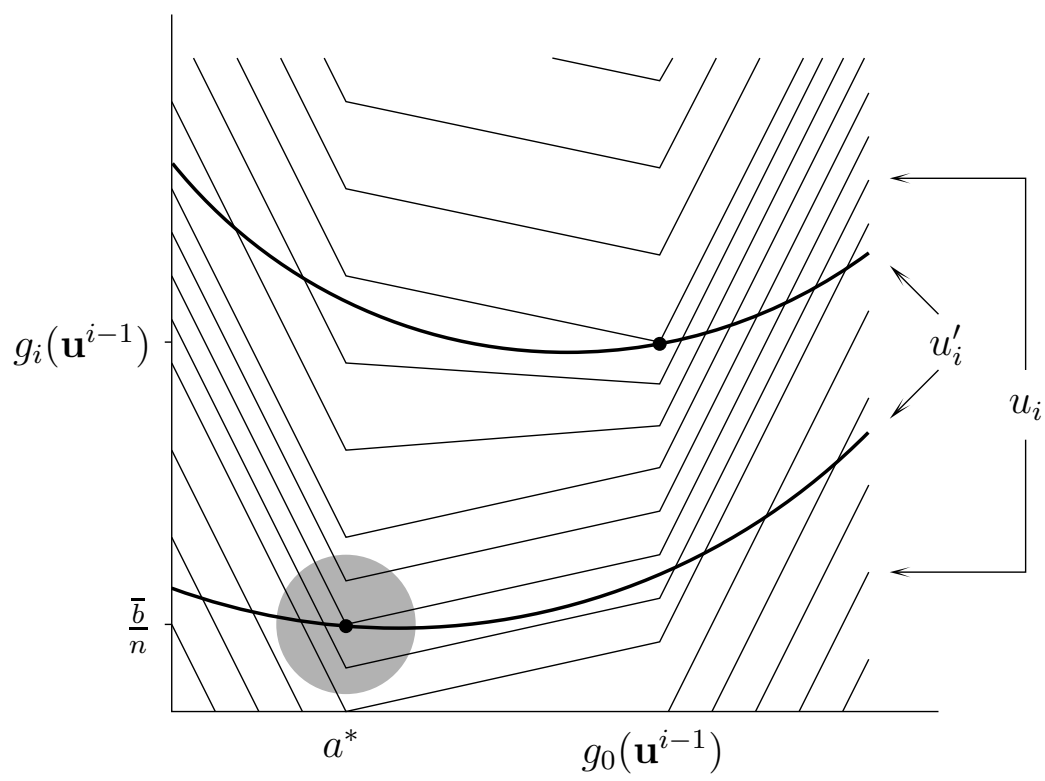

Figure 4: The function $u_{i}^{\prime}$ of Case 3. Area of local quasilinearity is shadowed

We now have the economy $\mathbf{u}^{\prime}$ which is in the domain $\mathcal{D}(\overline{\mathbf{u}}, f(\overline{\mathbf{u}}))$. For each $i, u_{i}^{\prime}$ is a sharpening of $u_{i}$ at $\left(a^{*}, \bar{b} / n\right)$, and $g_{0}\left(\mathbf{u}^{\prime}\right) \neq a^{*}$. By efficiency it is not the case that for all $i, u_{i}^{\prime}=u^{*}$ (see eq. (8)). Replace the utilities of those agents whose utility 
is not $u^{*}$ with $u^{*}$, one at a time. There is an agent $i$ such that replacing his utility $u_{i}^{\prime}$ with $u^{*}$ will change the quantity of the public good from $a^{\prime} \neq a^{*}$ to $a^{*}$. Denote the profile before $i$ makes the change $\mathbf{u}^{\prime \prime}$. Consider the path $\left\{u_{i}^{\alpha}:=\alpha u^{*}+(1-\alpha) u_{i}^{\prime}\right\}$ where $\alpha \in[0,1]$. Denote $\mathbf{u}^{\alpha}=\left(\mathbf{u}_{-i}^{\prime \prime}, u_{i}^{\alpha}\right)$. As both $u^{*}$ and $u_{i}^{\prime}$ are quasiconcave, and as each indifference curve of $u^{*}$ is a sharpening of an indifference curve of $u_{i}^{\prime}$ at a point along the line $a=a^{*}$, it follows that each element of the path is a quasiconcave function. Moreover, as both $u^{*}$ and $u_{i}^{\prime}$ are locally quasilinear around $\left(a^{*}, \bar{b} / n\right)$, so is each element of the path.

The point $a^{*}$ (together with equal allocation of the money commodity) is an efficient allocation for $\bar{u}$, as it is the outcome of the mechanism $f$ for that profile. Its efficiency does not disappear when utility functions are replaced with sharpenings at $\left(a^{*}, \bar{b} / n\right)$. Consider a profile $\tilde{\mathbf{u}}^{\alpha}$ where for every $j, \tilde{u}_{j}^{\alpha}$ is quasilinear, and shares with $u_{j}^{\alpha}$ the indifference curve through $\left(a^{*}, \bar{b} / n\right)$. It is well-known that for quasilinear utility, a necessary and condition for efficiency of $\left(a, b_{1}, \ldots, b_{n}\right)$ when each $b_{i}>0$ is that $\sum_{i} v_{i}\left(a^{*}\right)=\max _{a} \sum_{i} v_{i}(a)$. This, and Fact 1 imply that $\left(a^{*}, \bar{b} / n, \ldots, \bar{b} / n\right)$ is the only efficient IC outcome for $\tilde{\mathbf{u}}^{\alpha}$. But all the utility functions of the profile $\mathbf{u}^{\alpha}$ are locally quasilinear around $\left(a^{*}, \bar{b} / n, \ldots, \bar{b} / n\right)$, and moreover, for each $j$, there is a constant segment along the line $a=a^{*}$ around $\bar{b} / n$ that belongs to the quasilinear region. Therefore, there is a neighborhood of $\left(a^{*}, \bar{b} / n, \ldots, \bar{b} / n\right)$ where this point is the only efficient IC outcome for all $\alpha$. As this point is not the outcome of the mechanism for $\alpha=0$, but it is the outcome of the mechanism for $\alpha=1$, we obtain a contradiction. Therefore on the domain $\mathcal{D}(\overline{\mathbf{u}}, f(\overline{\mathbf{u}})), g_{0} \equiv a^{*}$, and by Fact 1 the private good is allocated equally to all, as in the mechanism $f$.

Proof of Corollary 1 Incentive compatibility of the mechanism follows from the fact that the mechanism on this domain is constant. Anonymity follows by the fact that everyone receives the same quantity of $b$. Next we prove efficiency. Define $L_{i}\left(a^{*}, a, b\right)$ to be the amount of private good compensation (positive or negative) person $i$ with the utility function $u_{i}$ will require to move from $a^{*}$ to $a$, given that he holds $b$ units of the private commodity. By the definition of $L_{i}$, if the economy shifts from $a^{*}$ to $a$, total compensation (in terms of the $b$ good) required to keep every one at the same utility level is $\sum_{i=1}^{n} L_{i}\left(a^{*}, a, \frac{\bar{b}}{n}\right)$ which is positive, hence $\left(a^{*}, \bar{b} / n, \ldots, \bar{b} / n\right)$ is efficient for $\mathbf{u}$.

Let $\mathbf{u}^{\prime} \in \mathcal{D}$ and suppose that person $i$ 's utility is not a sharpening of $u_{i}$ at $\left(a^{*}, \bar{b} / n\right)$. As the biggest change in $L_{i}$ person $i$ can obtain is from $-M$ to $M$ or from $M$ to $-M$, and as, by the definition of minimally comprehensive domains, the utilities $u_{j}^{\prime}$ of all other individuals $j$ are sharpenings of $u_{j}$ at $\left(a^{*}, \bar{b} / n\right)$, it follows by eq. (6) that 
$\left(a^{*}, \bar{b} / n, \ldots, \bar{b} / n\right)$ is still efficient.

Proof of Corollary 3 For $\mathbf{u}^{\prime} \in \mathcal{D}\left(\mathbf{u}, a^{*}\right)$, the mechanism is given by $f_{0}\left(\mathbf{u}^{\prime}\right)=a^{*}$ and $f_{1}\left(\mathbf{u}^{\prime}\right)=\cdots=f_{n}\left(\mathbf{u}^{\prime}\right)=\bar{b} / n$. Note that a profile $\mathbf{u}^{\prime}$ can satisfy inequality (6) at not more than one of the two points $\left(a^{*}, \bar{b} / n, \ldots, \bar{b} / n\right)$ and $\left(a^{* *}, \bar{b} / n, \ldots, \bar{b} / n\right)$. Therefore, if $\left(\mathbf{u}, a^{*}\right)$ and $\left(\overline{\mathbf{u}}, a^{* *}\right)$ satisfy $(6)$, then $a^{*} \neq a^{* *}$ implies $\mathcal{D}\left(\mathbf{u}, a^{*}\right) \cap \mathcal{D}\left(\overline{\mathbf{u}}, a^{* *}\right)=\varnothing$. Note that for all $\mathbf{u}^{\prime} \in \mathcal{D}\left(\mathbf{u}, a^{*}\right), f\left(\mathbf{u}^{\prime}\right)=a^{*}$ and for all $\mathbf{u}^{\prime} \in \mathcal{D}\left(\overline{\mathbf{u}}, a^{* *}\right), f\left(\mathbf{u}^{\prime}\right)=a^{* *}$. The domain $\mathcal{D}$ is therefore well-defined.

\section{References}

[1] Barbera, S. and M. Jackson, 1994. "A characterization of strategy-proof social choice functions for economies with pure public goods," Social Choice and Welfare, $11: 241-252$.

[2] Clarke, E.H., 1971. "Multipart pricing of public goods," Public Choice, 11:17-33.

[3] Groves, T. and M. Loeb, 1975. "Incentives and public inputs," Journal of Public Economics, 4:211-226.

[4] Hammond, P., 1979. "Straightforward individual incentive compatibility in large economies," Review of Economic Studies, 46:263-282.

[5] Jehiel, P., M. Meyer-Ter-Vehn, B. Moldovanu, and W.R. Zame, 2006. "The limits of ex post implementation," Econometrica, 74:585-610.

[6] Makowski, L and J. M. Ostroy, 1992. "Groves mechanisms in continuum economies: Characterization and existence," Journal of Mathematical Economics, $21: 1-35$.

[7] Makowski, L., J.M. Ostroy, and U. Segal, 1999. "Efficient incentive compatible economies are perfectly competitive," Journal of Economic Theory, 85:169-225.

[8] Maskin, E., 1999. "Nash Equilibrium and Welfare Optimality," Review of Economic Studies, 66: 23-38.

[9] Mitsui, T., 1983. "Asymptotic efficiency of the Pivot mechanism with a general project space," Journal of Economic Theory, 31:318-331.

[10] Moulin, H., 1980. "On strategy-proofness and single peakedness," Public Choice, 35: $437-455$. 
[11] Rob, R., 1982. "Asymptotic efficiency of the demand revealing mechanism," Journal of Economic Theory, 28:209-220.

[12] Samuelson, P., 1954. "The Pure Theory of Public Expenditure," The Review of Economics and Statistics, 387-389.

[13] Serizawa, S., 1999. "Strategy-proof and symmetric social choice functions for public good economies," Econometrica, 67:121-145.

[14] Schummer, J., 2000. "Manipulation through Bribes," Journal of Economic Theory, 91:180-198.

[15] W. Vickrey, W., 1961. "Counterspeculation, auctions, and competitive sealed tenders," Journal of Finance, 16:8-37.

[16] Walker, M., 1980. "On the nonexistence of a dominant-strategy mechanism for making optimal public decisions," Econometrica, 48:1521-1540.

[17] Zhou, L., 1994. "Impossibility of strategy-proof mechanisms in economies with pure public goods," Review of Economic Studies, 58:107-119. 